\title{
Visual factors and dyslexia: A research review
}

\author{
Wajuihian SO* and Naidoo KS $\mathrm{K}^{\dagger}$
}

*African Vision Research Institute (AVRI) University of KwaZulu-Natal, Private Bag X54001, Durban, 4000 South Africa.

\section{† International Center for Eye Care Education, 172 Umbilo Road, Durban 4001, South Africa}

\begin{abstract}
Eye care professionals have long been involved with vision and learning, and receive referrals from teachers, psychologists and other professionals who seek advice about whether vision problems may contribute to, or be responsible for, poor academic performance. This is particularly the case when dealing with the relationship between vision and dyslexia, which has been a subject of controversy in optometric, ophthalmologic, educational and psychological literature. Ophthalmologists and
\end{abstract}

optometrists in particular, hold different views on the association of dyslexia and vision, and there has been much debate regarding any causal relationship between them. The results of studies that investigated the vision functions in dyslexic populations remain inconclusive. This review is intended to provide an overview of studies to date and makes recommendation to guide future research. ( $S$ Afr Optom 2010 69(2) 58-68)

Key words: Dyslexia, learning disabilities, vision, review of visual factors in dyslexia

\section{Introduction}

The term dyslexia is derived from the Greek words: 'dys' meaning hard or difficult, and 'lexia' from the word 'lexicos' which means 'pertaining to words'. Dyslexia therefore means a difficulty with words ${ }^{1}$.

As documented by Christenson et al ${ }^{3}$, the term dyslexia was first introduced by a German Physician, Berlin in 1887. In 1968, the World Federation of Neurologists ${ }^{1}$ defined dyslexia as "a disorder manifested by difficulty in learning to read despite conventional instruction, adequate intelligence and socio-cultural opportunity. It is dependent upon fundamental cognitive disabilities which are frequently constitutional in origin".

Reading, which is the process of deriving meaning from written text, is a fundamental aspect of learning. Learning to read involves a complex system of skills relevant to visual (the appearance of a word), ortho- graphic (visual word form), phonological (processing the distinctive sound elements that constitutes a word), and semantics (meaning of words and phrases) processing. Reading also involves a variety of behaviors: letter naming, letter perception, word recognition and comprehension, each of which uses a different part of the brain. For some children, reading becomes difficult due to their inability to break words into phonemes (smallest component units of a word) 3,4 .

Dyslexia is the most common and carefully studied type of learning disability, affecting $80 \%$ of all those identified as learning-disabled ${ }^{3}, 5$. There is a large variation in the prevalence of dyslexia reported, but estimates of the prevalence in Caucasian populations range between 1 and $20 \%{ }^{6-8}$. Park ${ }^{9}$ documented that up to $15 \%$ of the school-age population has some degree of dyslexia. The large variability in the prevalence reported by different authorities may be attributed to the differences in the diagnostic crite-

${ }^{*}$ BSc(Hons) Optom OD(Benin) MOptom (UKZN) PGCertMod L/Vision(City Univ London) †BSc BOptom(UDW) MPH (Temple) OD (PCO) PhD (UNSW) 
ria and the cut-off point applied to the psychometric tests $^{10-12}$.

Dyslexia is a syndrome ${ }^{13}$ whereby dyslexic individuals experience a range of symptoms which may include: difficulty learning to read, word reversal, instability of binocular fixation, skipping complete words while reading, double vision, faulty pronunciation, the text jumping around on a page, letters of some words appearing completely backwards such as the word bird looking like $d r i b$, or the inability to tell difference between letters: $o$ and $e$, or $b, p$, and $q$ 6,14 . These symptoms result in the person being unable to link words to make a sentence and therefore to comprehend the meaning of the sentence. As a result, they have difficulty in school and in areas of life that require the use of words, as reading is important for the academic, economic, and social success of a person.

The relationship between vision and dyslexia has been a subject of controversy in optometric ${ }^{15-18}$ and ophthalmological literature ${ }^{19-24}$. The studies on dyslexia by eye professions are few but they all contribute to a better understanding of possible associations between dyslexia and eye problems. One of the earliest studies on the subject of vision and reading difficulty was conducted by Eames ${ }^{25}$ in 1948, in which he described the participants as being reading disabled. Studies ${ }^{15-24}$ on the relationship between vision and dyslexia do not establish a causal relationship between vision and dyslexia, but tend to establish associations between dyslexia and certain vision variables. The literature also indicates that optometry and ophthalmology hold different views on the likely association between dyslexia and vision. Most studies from the optometric domain maintain that vision may not be considered a cause of dyslexia, but that compensating for vision defects makes reading easier for the dyslexic child ${ }^{15-18}$. However, a contrary view was expressed by ophthalmologists as according to Helveston et al19 "some optometrists, psychologists, and teachers assign a significant role to the eyes as a cause of reading failure. This is in contrast to most ophthalmologists who deny that any role is played by the eyes and visual functions in the cognitive aspect of reading". Helveston et al ${ }^{19}$ failed to supply a scientific study to support their claim.

These conflicting views between ophthalmology and optometry have been further emphasized in the
Policy Statements of American Academy of Pediatrics, the American Academy of Ophthalmology and American Association for Pediatric Ophthalmology and Strabismus which states ${ }^{26}$. "there is inadequate scientific evidence to support the view that subtle eye or visual problems, including abnormal focusing, jerky eye movements, misaligned or crossed eyes, binocular dysfunction, visual-motor dysfunction, visual perceptual difficulties... cause learning disabilities". "Ineffective, controversial methods of treatment such as vision therapy may give parents and teachers a false sense of security that a child's learning difficulties are being addressed, may waste family and/or school resources, and may delay proper instruction or remediation". Because visual problems do not underlie dyslexia, approaches designed to improve visual function by training are misdirected".

The view of optometry however, is that optometrists do not treat dyslexia or learning disability but treat visual conditions that would constitute an impediment to educational remediation ${ }^{27}$. Hence, according to the joint organizational policy statement of the American Academy of Optometry and the American Optometric Association"27 "The expected outcome of optometric intervention is an improvement in visual function with the alleviation of associated signs and symptoms. Optometric intervention for people with learning-related vision problems consists of lenses, prisms, and vision therapy. Vision therapy does not directly treat learning disabilities or dyslexia. Vision therapy is a treatment to improve visual efficiency and visual processing, thereby allowing the person to be more responsive to educational instruction. Such therapy does not preclude other forms of treatment and should be a part of a multidisciplinary approach to learning disabilities."

Another aspect of the controversy is the inconsistency in the results reported by different authors. Various studies ${ }^{15-24}$ have examined several aspects of vision suspected of affecting reading ability. However, the major drawbacks that hinder such research are:

- Intelligence quotient is an important factor to consider when defining dyslexia. Only a few studies have considered intelligence quotients in their subjects' selection.

- Some studies did not include a control group which made the reports difficult to assess.

- Some researchers did not classify terms such as bin- 
ocular coordination and refractive errors, making it difficult to evaluate the impact of a particular vision variable on reading ability.

- Some studies did not indicate the eye and vision examination techniques used in their protocol which made a comparison and the replication of such studies difficult.

\section{Literature Review}

It is useful to present an update on studies conducted to investigate vision functions in dyslexic populations and offer a research perspective that will guide future research in dyslexia and vision. The visual functions reviewed include visual acuity, refractive errors, near point of convergence, ocular alignment, accommodation functions, vergences and reserves.

\section{Visual Acuity (VA)}

Vision plays an important role in the reading process, as the ability to see printed material is essential for reading. A marked bilateral reduction in VA can therefore limit reading performance ${ }^{28}$. The assessment of visual acuity in dyslexic children is an important test, as it detects visual problems at distances at which most school learning activities occur, and gives useful preliminary information on the state of a child's vision. Uncorrected visual acuity may impair learning capability and educational potential in school children and could interfere with the development of the visual pathways if left untreated ${ }^{29}$. A reduced visual acuity could be due to an ocular abnormality (such as cataract) or to normal refractive error differences in the population. Visual acuity defects due to ocular diseases are usually not common in the paediatric population ${ }^{30}$ but tend to be more related to refractive error changes ${ }^{31}$.

The literature reveals that the findings on visual acuity defects in dyslexic children are diverse. In 1994, Latvala et al20 assessed the ophthalmic status of 55 dyslexic and 50 control subjects in an age, gender and social class matched study in Finland. The subjects' school grades and intelligence quotient were not indicated. The authors assessed visual acuity using the Snellen chart, and found that for the control group, all subjects had visual acuity of 0.7 (6/9), while in the dyslexic group, two children $(3.6 \%)$ had bilateral visual acuity of less than or equal to 0.7 (6/9).
A study to examine the ophthalmological status of school children with dyslexia in Norway was conducted by Aasved ${ }^{21}$ in 1987. Vision examinations were performed on 259 dyslexic children. The author reported that the dyslexic population had a higher prevalence of reduced visual acuity than the normal readers, and it was concluded that although there was no causal relationship between eye characteristics and reading difficulties, eye abnormalities should be corrected when detected in dyslexic children.

In 1993, Evans et al ${ }^{15}$ studied sensory and refractive visual factors in 39 dyslexic and 43 control subjects aged between 7 years 6 months and 12 years 3 months in the United Kingdom. Participants from both groups had intelligence quotients of above 85 . VA was measured using the LogMAR Chart. The authors reported that although the dyslexic group had a significantly worse near and distance binocular VA, there were no statistically significant differences between the two groups, (binocular distance VA, $p=0.0164$ and binocular near VA, $p=0.0018$ ).

In a comprehensive study conducted in 2007 by Kapoula et $a l^{22}$, convergence and divergence functions in 57 dyslexic and 46 non-dyslexic age-matched French children were investigated. For the dyslexic subjects, the mean age was 11.3 years, the mean IQ was 105 and the mean reading age was 8.91. For the control group, the mean age was 10.7 years while their IQ score was not indicated. The details of the technique used to assess visual acuity was not given although the measurement technique for near point of convergence and fusional reserves was given. It was reported that all participants had normal visual acuity of $6 / 6$.

Buccis et $a l^{23}$ in 2008 studied poor binocular coordination of saccades in dyslexic and non-dyslexic children in France. The vision functions assessed were VA, refraction (performed under cycloplegia), heterophoria, near point of convergence and fusional vergence reserves. The study participants comprised 18 dyslexic children of mean age 11.4 years and mean IQ of 105, with aged-matched non-dyslexic children, mean age 11.2 years. There were no details on the method used to assess VA and refraction, but it was indicated that a cycloplegic refraction was performed. The authors reported that all children had normal VA of $6 / 6$.

In 1991, a study on stereopsis, accommodative and 
vergence facility was conducted by Buzzelli ${ }^{16}$ in the United States of America. Thirteen normal readers of average age 13 years 4 months, and 13 dyslexics with an average age of 13 years 3 months participated in the study. Both groups were matched for gender, age and intelligence quotient. The study design was comprehensive, and the Snellen chart was used to assess the VA. Buzzelli ${ }^{16}$ found that the dyslexics did not perform worse than the control subjects in VA but the $p$-value was not indicated for the visual acuity results.

A study conducted by Ygge et al ${ }^{24}$ in 1992 studied visual functions in a Swedish population of dyslexic and normally reading children. The participants consisted of 86 nine-year-old children who were matched to controls with regard to age, gender, class in school and intelligence. VA was assessed with 'ordinary optotypes charts'. They reported that the subjects from the control group had a better visual acuity than the dyslexic group at both distance and near, and that the results showed statistically significant differences (distance at $p=0.03$ and near at $p=0.005$ ).

A comparative study using orthoptic assessment procedures was conducted by Goulandris et $a l^{17}$ in the United Kingdom in 1998. This study consisted of 20 dyslexic and 20 chronological and reading agematched controls; a unique aspect of the study being that the distance VA was assessed using the Cambridge Crowding Cards. This test, according to the authors, was devised to evaluate letter recognition acuity in conditions of 'crowding' when other letters surround the target letter and to ensure that abnormalities of vision, which would not be revealed reliably using single targets, were detected. The near acuity was assessed using the Snellen chart. The use of different visual acuity charts at distance and near raises a concern of standardization and also limits comparisons between studies. The prevalence of distance and near VA (using a 6/9 criteria) was reported to be similar between the groups.

Castro et al $^{14}$, in 2008 , studied visual functions in 13 dyslexic and 13 control subjects in Brazil. The mean age for the dyslexic and control participants was 11 years 2 months. They reported that all participants had visual acuity of 6/6 at both distance and near except one participant from the dyslexic group who was anisometropic and had VA of 6/9 at both distance, and near.
It is evident from the results of these studies that it cannot be concluded that there are significant differences in uncompensated visual acuity between dyslexic and non-dyslexic children.

\section{Refractive Errors (RE)}

Some studies classified RE according to the type (myopia, hyperopia, astigmatism) while others did not. Amongst the studies that did not classify RE was the study by Evans et al ${ }^{15}$, the details of which were presented earlier. Refractive error was assessed using the streak retinoscopy technique and the distribution of RE was found to be similar in both groups. The use of subjective data for the study analysis may be problematic due to the possible influence of factors such as participants' fatigue and malingering. Similar to Evans et al ${ }^{15}$, Ygge et al ${ }^{24}$ assessed RE objectively using streak retinoscopy in cycloplegia and reported that the distribution of RE was similar between the two groups in their study.

The reports ${ }^{15,24}$ on myopia were classified broadly as refractive error which made it difficult to analyze the findings on how myopia (specifically) is related to reading ability. But hyperopia is often associated with poor reading performance, probably through the mechanism of deficient accommodation and poor motor fusion skills ${ }^{28}$. In relating hyperopia to reading difficulties, simple to moderate hyperopia may not cause constant blur at a distance or near point, but the extra accommodative effort produces asthenopic symptoms of intermittent blur, headache, fatigue, loss of concentration and inattention in some patients, which may be mistaken for a short attention span. Uncompensated hyperopia is associated with esophoria at the near point, which can stress the fusional vergence systems that hold the eyes in correct alignment ${ }^{32}$. If the hyperopia and esophoria is excessive, an accommodative esotropia can result ${ }^{32}$. In the refractive error studies reviewed ${ }^{15,20,24}$ the prevalence of hyperopia was classified as refractive error ${ }^{15,24}$ which made it difficult to analyze the findings on how hyperopia (specifically) affects reading performance.

The following studies presented refractive errors independently as astigmatism, amblyopia or anisometropia. Astigmatism is an important clinical and public health concern because, in contrast to other refractive error states, a child with uncompensated 
astigmatism experiences blur on a continuous basis. Secondly, an astigmatic child is at increased risk for the development of refractive amblyopia. Furthermore, optical blur imposed by astigmatism may predispose to myopia development ${ }^{33}$. In a study by Latvala et al 20 , the prevalence of astigmatism (defined as greater than $-1.00 \mathrm{D}$ cylinder) was higher in the dyslexic group $(3.6 \%)$ as compared to the control group (none). Ygge et al ${ }^{24}$ also reported that the prevalence of astigmatism was higher in the dyslexic group (right eye, 28\% and left eye, $25 \%$ ) compared to the control group (right eye $18.3 \%$ and left eye $24.3 \%$ ) although there was no statistically significant difference between the groups $(p=0.25)$.

The only available study that reported on the prevalence of amblyopia was by Latvala et al ${ }^{20}$, which reported the prevalence of amblyopia in the dyslexic group to be $3.6 \%$, while no participant in the control group had amblyopia.

Anisometropia, a difference in refractive error between two eyes, is of great clinical interest due to its intimate association with strabismus and amblyopia ${ }^{34,35}$. Generally, anisometropia may be defined as a difference of $1 \mathrm{D}$ or more of refraction between the two eyes $^{21}$. Uncorrected anisometropia, even of moderate amount, may induce eye strain as it is impossible for the accommodation mechanism to maintain clear images on the retina at the same time. However, large amounts of anisometropia (2 D or more) may not cause symptoms, as no effort is made to maintain single binocular vision ${ }^{36}$. Latvala et al ${ }^{20}$ reported a higher prevalence of anisometropia (of $\geq 1 \mathrm{D}$ diopter) of $6 \%$ in the control group than in the dyslexic group $3.6 \%$. Similarly, Ygge et al 24 found a higher prevalence of anisometropia in the control group (15.8\%) than in the dyslexic group (9.4\%).

\section{Near Point of Convergence (NPC)}

Convergence is the simultaneous inward movement of both eyes toward each other, usually in an effort to maintain single binocular vision when viewing an object at near, and is a type of inward vergence eye movement ${ }^{37}$. The process of reading requires sustained convergence ${ }^{38}$. A study by Owens and WolfKelly ${ }^{39}$ concluded that "near work induces a recession of the near point of accommodation or vergences".
Kapoula et al 22 assessed the NPC by placing a small pen-light at 30-40 cm in the mid-plane in front of the subject, and moving it slowly towards the eyes until one eye lost fixation. The authors reported that the NPC was significantly more remote $(>10 \mathrm{~cm})$ in dyslexics $(36 \%)$ than in non-dyslexics $(15 \%)$. Buccis et al ${ }^{23}$ followed the same testing procedure as in the study by Kapoula et al 22 and reported that the NPC was not statistically different between the two populations. The technique used in assessing the near point of convergence was not indicated in the study by Latvala et $a l^{20}$ but this study reported that convergence near point greater than or equal to $8 \mathrm{~cm}$ was higher $(12.7 \%)$ for the dyslexic subjects than in that for the control group $(2 \%)$. The authors stated that the NPC was the only variable that showed a statistically significant difference ( $p=0.0385$ ) between the groups, and concluded that ophthalmic factors should not be overlooked as a contributing factor to dyslexia as they may constitute part of the dyslexic syndrome.

A study by Evans et al ${ }^{18}$ conducted in the United Kingdom in 1993 investigated accommodative and binocular functions in dyslexia. The subjects were 43 control and 39 dyslexics aged between 7 years 6 months and 12 years three months. An intelligence quotient above 85 for the dyslexics and above 90 for the control, using the Wechsler Intelligence Scale for Children (Revised) (WISC-R), was reported. They measured NPC using the royal air force (RAF) rule, and there was no statistically significant difference between the two groups in the NPC test $(p=0.54)$.

\section{Heterophoria}

The presence of heterophoria could contribute to visual and attention abnormalities noted among children with reading difficulties, making near point visual activities more strenuous. Furthermore, refractive error, fusional vergences and heterophoria are interrelated as an uncorrected hyperopia is associated with esophoria at near point which can stress the fusional vergence systems that hold the eyes in correct alignment. If the hyperopia is associated with a high AC/A ratio, an accommodative esotropia can result ${ }^{32}$.

As shown in Table 1 the findings on heterophoria in dyslexic populations are diverse. 
Table 1. Summary of heterophoria results from different studies.

\begin{tabular}{|c|c|c|c|c|c|}
\hline & & & Dyslexia & Control & \\
\hline Author(s) & $\begin{array}{c}\text { Variable } \\
\text { (Heterophoria) }\end{array}$ & $\begin{array}{l}\text { Measurement } \\
\text { Techniques }\end{array}$ & Results & & Comments \\
\hline $\begin{array}{l}\text { Latvala } \\
\text { et } \text { d }^{20}\end{array}$ & $\begin{array}{l}\text { At distance } \\
\text { At near }\end{array}$ & $\begin{array}{l}\text { Cover test } \\
\text { Cover test, } \\
\text { Maddox wing }\end{array}$ & $\begin{array}{l}\text { esophoria } 1.3 \% \\
\text { exophoria } 1.9 \% \\
\text { vertical phoria } 3.8 \% \\
\\
\text { exophoria } 5.8 \% \\
\text { exophoria } 25 \% \\
\text { vertical phoria } 3.6 \%\end{array}$ & $\begin{array}{l}\text { esophoria } 8.2 \% \\
\text { exophoria } 2 \% \\
\text { vertical phoria } 4.1 \% \\
\\
\text { esophoria } 6.1 \% \\
\text { exophoria } 2.2 \% \\
\text { vertical phoria } 6.1 \%\end{array}$ & $\begin{array}{l}\text { No statistically significant } \\
\text { difference between the two } \\
\text { groups }(p=0.59) \text { for } \\
\text { exophoria and }(p=0.46) \text { for } \\
\text { exophoria }\end{array}$ \\
\hline Buccis et $a l^{23}$ & $\begin{array}{l}\text { At distance and } \\
\text { near }\end{array}$ & $\begin{array}{l}\text { Cover test and } \\
\text { the Maddox rod }\end{array}$ & & & $\begin{array}{l}\text { No statistically significant } \\
\text { difference between the two } \\
\text { groups }(p=0.2) \text {. }\end{array}$ \\
\hline Evans et $a l^{18}$ & $\begin{array}{l}\text { At distance } \\
\text { At near, }\end{array}$ & $\begin{array}{l}\text { Cover test, } \\
\text { Maddox Wing }\end{array}$ & $\begin{array}{l}\text { all subjects were } \\
\text { orthophoric }\end{array}$ & $\begin{array}{l}\text { all subjects were } \\
\text { orthophoric }\end{array}$ & $\begin{array}{l}\text { Horizontal phoria results } \\
\text { were similar in both group }\end{array}$ \\
\hline $\begin{array}{l}\text { Ygge et } \\
a l\end{array}$ & At distance & Cover test & $\begin{array}{l}9 \text { subjects had eso- } \\
\text { and } 14 \text { had exo- } \\
\text { phoria }\end{array}$ & $\begin{array}{l}6 \text { eso- and } 14 \text { exo- } \\
\text { phoria }\end{array}$ & $\begin{array}{l}\text { No statistically significant } \\
\text { difference }(p=0.34) \text {. }\end{array}$ \\
\hline
\end{tabular}

In another report, Ygge et al 40 studied oculomotor functions in a Swedish population of dyslexic and non-dyslexic children. The study comprised 86 nine year old dyslexic children matched to control with regard to age, gender and intelligence. Heterophoria was measured using the cover test. They reported that $26 \%$ of the participants from the dyslexic group had heterophoria whereas in the control group $23.2 \%$ had heterophoria and that the difference was not statistically significant with the $p$-value not indicated. At near, the incidence of exophoria was $43.7 \%$ for the dyslexic group and $45.3 \%$ in the control group. There was no statistically significant difference. The $p$-value was not clearly indicated.

The assessment of heterophoria was comprehensive in the study conducted by Latvala et al ${ }^{20}$. They measured heterophoria using the cover test and prism bars to quantify the findings. At near, they report- ed a higher prevalence of esophoria (6.1\%) among the control than the dyslexic group (5.8\%) a higher prevalence of exophoria (25\%) among dyslexics than the control group $(12.2 \%)$ and a higher prevalence of vertical phoria $(6.1 \%)$ among the control than the dyslexic subjects $(3.6 \%)$. At distance, they found a higher incidence of esophoria (11.3\%) among dyslexics than the control $(8.2 \%)$ subjects, a higher prevalence of exophoria (2\%) among the control group than among the dyslexics $(1.9 \%)$ and vertical phoria to be more prevalent in the control $(4.1 \%)$ than in the dyslexics $(3.8 \%)$. There was no statistically significant difference between the two groups $(p=0.59)$ for exophoria and ( $p=0.46$ ) for esophoria. Evans et al ${ }^{18}$, in a study of accommodative and binocular functions in 43 control and 39 dyslexics, reported that all subjects in the examined group were orthophoric at distance with cover test, except for one dyslexic subject. At 
near, horizontal phoria results were similar in both groups. The Maddox wing was used to assess near phoria by Evans et al ${ }^{18}$. Buccis et al ${ }^{23}$ measured heterophoria at far and near using the cover-uncover test and found no statistically different between the two groups $(p=0.2)$.

Castro et al ${ }^{14}$ asessed heterophoria using the cover test and reported that only one participant from the dyslexic group presented with exophoria at a distance of 4 meters of 6 prism diopters (pd) and at 33 centimeters, three participants in the dyslexic group had exophoria of $6 \mathrm{pd}$ and two had exophorias from 10 to $12 \mathrm{pd}$.

\section{Strabismus}

Several authors included the measurement of strabismus in their studies, reporting remarkably varying findings. In the study by Latvala et al ${ }^{20}$ the prevalence of tropia at near for the dyslexic group was 3.6\% and $2 \%$ for the control group. The cover test was used to assess ocular alignment. Aasved ${ }^{21}$ assessed ocular alignment using the cover test and the prism bar to quantify the deviations, and found that the dyslexics had a higher prevalence of manifest and latent convergent strabismus than the control subjects. The statistical significance ( $p$-value) was not indicated. Goulandris et al ${ }^{17}$ measured strabismus using the cover test and found no statistically significant difference between the dyslexic and control groups in the prevalence of strabismus $\left[\chi^{2}(2)=1.03\right.$, ns]. In contrast, Ygge et al ${ }^{40}$ reported that at distance manifest strabismus was more frequent among the dyslexic (8\%) pupils than the control (2.3\%), but that the difference was not statistically significant. At near, the prevalence of manifest strabismus was $8 \%$ (all eso) for the dyslexics and 3.5\% (all eso) in the control group. The $p$-values were not indicated.

\section{Accommodative Functions}

The accommodation mechanism is extremely important for learning. Children who suffer some anomalies of accommodation are prone to more fatigue and are inattentive sooner than those who have normal accommodation function ${ }^{41}$. The findings on various aspects of accommodation functions were diverse.

Amplitude of Accommodation (AA)

Accommodation is the ability to adjust the focus of the eye by changing the shape of the crystalline lens to attain maximum sharpness of the retinal image of an object of regard. The absolute magnitude of the accommodative response is termed the accommodative amplitude ${ }^{42}$. Evans et al ${ }^{18}$ measured AA using the push-up method and reported that AA was significantly reduced in the dyslexic group. In the study by Helveston et al ${ }^{19}$, the AA was measured using the push up to blur technique. It was reported "an ability to accommodate more than eleven diopters was found in between $90 \%$ and $93 \%$ of the students" that participated in the study. Ygge et al ${ }^{40}$ assessed AA using the RAF rule and reported that the two groups performed similarly. A similar technique was used by Goulandris et al ${ }^{17}$ who found no statistically significant difference between the groups.

\section{Accommodation Facility (AF)}

Accommodative facility assesses the rate at which accommodation can be stimulated and inhibited repeatedly during a specific time period. Accommodation facility relates to the individual's ability to shift focus quickly and efficiently for varying distances and is used extensively in the reading process ${ }^{16}$. Evans et al ${ }^{18}$ assessed AF using a technique they described as a "more realistic test which measured the rate at which subjects could change their vergence and accommodation between distance $(3 \mathrm{~m})$ and near $(30 \mathrm{~cm})$ ". It was reported that the dyslexic group appeared to be slower at a test of accommodative facility. Buzzelli ${ }^{16}$ found that the dyslexic subjects showed better AF than the control group, although there was no statistically significant relationship between the two groups ( $p=0.629)$ for binocular accommodation facility. It should be noted that when evaluating AF, it is important to note the difficulty and speed of responses to the positive and negative lenses, and whether the subjects fatigue easily ${ }^{43}$ as this could affect the outcome of the comparison.

In a review conducted by Evans and Drasdo ${ }^{44}$ in 1990, convergence insufficiency and accommodative dysfunctions were reported to be more common in a dyslexic population than in normal readers.

\section{Accommodation Lag (AL)}

During near vision, the eyes are not usually precisely focussed on the object of regard, but the accommodation lags slightly behind the target. If the 
accommodative lag is low, then the blur it causes is insignificant; if it is high, then it can result in blurred print during reading ${ }^{30}$. Evans et al ${ }^{18}$ assessed AL using the monocular estimate method of retinoscopy and reported no statistically significant difference between the dyslexic and the control groups ( $p>0.68$ unpaired $t$-test). This was the only available study that reported on accommodation lag.

\section{Vergence Facility}

Vergences are a clinical measure of an individual's ability to overcome the prismatically induced optical displacements of a target in space. Convergence relates to each eye fixating closer in space than the real physical location of the target, while divergence is the measure of the eyes ability to fixate farther in space while still maintaining single binocular vision ${ }^{45}$. The only available study that reported on vergence facility was conducted by Buzzelli ${ }^{16}$. He reported that the dyslexics completed the vergence eye movement task significantly slower (300 seconds) than the normal readers (240 seconds). A statistically significant relationship between the groups was reported $(p<0.05)$. It was recommended that the possible role of vergence in dyslexia should be investigated on a larger dyslexic and control groups. From the literature reviewed, this was the only study that reported on vergence facility.

\section{Fusional Reserves (FR) and Vergence Amplitude}

Several authors $17-19,22,23,40$ included the assessment of vergence reserves using the prism bar in their study. However, the results are very diverse. The major findings were reported by Evans et al ${ }^{18}$, who found that fusional reserves were significantly reduced in the dyslexic group relative to the control. At near, for negative vergence reserves $(p=0.014,0.06$ and 0.08 for blur, break and recovery respectively) and for the positive vergence reserves, $(p=0.05,0.03$ and 0.088 for blur, break and recovery respectively $)^{18}$.

The incidence of fusional amplitude greater than or equal to 32 prism diopters at near was higher in dyslexic (7.5\%) than in control (6.1\%) group as reported by Latvala et al ${ }^{20}$. At distance, they found fusional amplitude greater than or equal to 15 prism diopters to be higher in the control $(12.2 \%)$ than in the dyslexic (9.4\%) group. Kapoula et al 22 measured vergence reserves using prism bars at far and near distance for both groups. For negative fusional vergence, it was reported that "divergence amplitude" was significantly different in the two groups for both distances $(p<0.005)$ and they concluded that vergence deficits are frequently present in dyslexics.

Buccis et al ${ }^{23}$ measured fusional reserves at far and near using prism bars (base in and base out). According to the authors, orthoptic evaluation of vergence fusion capability showed a significantly limited divergence capability for dyslexics compared to non-dyslexics $(p<0.001)$. In contrast, convergence amplitude was in the normal range for the two populations, even if for dyslexics the amplitude was significantly larger than for non-dyslexics $(p<0.01)$.

Ygge et al ${ }^{40}$ assessed vergence fusion using the prism bar. They reported that the fusion convergence and divergence capacities at distance and near were similar in the two groups. The mean fusional convergence capacities at distance were 16.8 prism diopters (pd) for both the control and the dyslexic group. At near, the corresponding figures were $26.4 \mathrm{pd}$ and 26.7 pd respectively. The mean fusional divergence capacity at distance was $6.5 \mathrm{pd}$ and $6.2 \mathrm{pd}$ in the dyslexic and control groups respectively, whereas at near the fusion divergence capacity was 10.5 and $10.2 \mathrm{pd}$ respectively. There was no statistically significant difference between the groups mean convergence fusion $(p=0.61)$, mean divergence fusion $(p=0.74)$ and mean total fusional amplitude $(p=0.55)$.

In 1988, Stein et al 46 investigated disordered vergence control in 39 dyslexic children aged 8 to 11 years, and a control group of 20 subjects of similar age in the United Kingdom. The vergence control was measured using the synoptophore and they reported that two thirds of the dyslexic group had abnormal vergence control. According to the authors, the study was the first in which eye movement recording was used to demonstrate differences between dyslexics and normal readers.

Assessing FR in children with loose prism bars may be a better technique as it offers the possibility of viewing the eye movement objectively. Secondly, it removes the restriction imposed by testing children behind the phoropter. However, it is important to note that most children have difficulty maintaining fixation long enough to measure vergence ranges. Another important aspect of the measurement of FR is the use of suppression control. Assessing fusional reserves without controlling for suppression may result 
in high break values; when suppression is controlled, the average vergence values may be lower as the test is stopped when the suppression is detected. If suppression is not monitored, the break is not detected until the stimulus is outside the suppression zone and a higher vergence value is obtained ${ }^{47}$.

\section{Discussion}

The findings reported by different authors are diverse. However, all the studies reviewed followed similar patterns with regard to their study design. The authors either compared the prevalence of vision defects in a population of dyslexic children to the prevalence of similar vision defects in a group of normal readers, or only presented the prevalence of a particular visual condition in a group of dyslexic children.

The findings on the relationship between vision and reading performance vary and remain inconclusive, making a comparative analysis difficult. The differing results may be due to the methodological differences or from differences in instrumentation and techniques, classification criteria, data analysis, lack of comparison group or the type of population studied (clinical/non-clinical). The fact that research on dyslexia and vision requires an interdisciplinary research approach with different professions having different perspectives in their investigation techniques/approach may also contribute to the diverse results reported.

Vision and reading performance are complex activities, and their measurements can be influenced by many factors ${ }^{13,28}$. Consequently, in evaluating the literature on reading disability and vision, it is important to note that it may be a challenge to design a study that would control for all variables that could influence reading ability and vision function. Flax ${ }^{13}$ shares similar views, and stated that "even in studies where there has been rigorous research design and control there has likewise been a failure for a stronger relationship to appear".

Uncorrected vision defects make reading and learning difficult, but a review of the literature suggests that defects of the visual system may not be considered a cause of dyslexia. Flax ${ }^{41}$ proposed that the role of visual factors in dyslexia depends on the definition of vision that is considered, emphasizing that if vision is narrowly defined as visual acuity or clarity of sight, it is unlikely that there would be any relationship between vision and dyslexia. If other peripheral visual functions such as fusion, convergence, refractive errors and accommodation are considered, there is likely to be a relationship, but then questions the possibility of vision being a causative factor.

Vision anomalies are contributory, and frequently hinder responses to educational intervention. In cases where vision anomalies are factors involved in the reading disability, the effort required to maintain clear, single binocular vision decreases the efficient organization of the visual cognitive response ${ }^{32}$. Vision difficulties are therefore only a part of the dyslexic syndrome ${ }^{13}$.

\section{Recommendations}

This review provided an analysis of studies on dyslexia and in the process, indicated areas of research needs. The following recommendations should be considered for future research.

1. The intelligence quotient of the study participants should be considered as it is crucial in the definition and classification of dyslexia.

2. It is important to properly detail all testing protocols to enable replication of the study by other researchers.

3. Studies with matched controls may be easier to analyse and compare with other studies than studies without matched control.

4. For easier comparison with other studies, it is recommended that vision variables such as refractive error and binocular anomalies be categorized by type.

5. Larger sample size using random sampling may yield more conclusive results.

6. Assessment of binocular functions should be performed with suppression control.

\section{Conclusion}

It can be concluded from the reported studies that the findings on dyslexia and vision are inconsistent, 
and that while some vision defects appeared more prevalent in the dyslexic population than the control subjects, there was no indication that the dyslexic children are exceptionally at risk of any vision condition. It is however recommended that for children to perform maximally any vision defects should be detected early and compensated for accordingly.

Despite the inherit limitations of previous studies, they provide a comprehensive overview of the possible associations of dyslexia and visual factors. Eye care professionals, educators and other professionals who work with dyslexic children may find it useful to have an overview of conditions that have been researched with regard to their impact on dyslexia. This will assist in their ability to make informed decisions.

\section{Acknowledgement}

We are grateful to Vanessa Moodley, Carrin Martin and Diane Wallace for commenting on the manuscript.

\section{References}

1. Krupska M, Klein C. Demystifying Dyslexia. London Language and Literacy Unit Publication, London 1995.

2. Christenson GN, Griffin JR, Wesson MD. Optometry's role in learning disabilities: Resolving the controversy. Journal of American Optometric Association 199061 (5) 363-372.

3. Shaywitz SE. Dyslexia. New England Journal of Medicine 1998338 307-312.

4. Tan L, Spinks JA, Eden GF, Perfetti CA, Siok WT. Reading depends on writing in Chinese. Proceedings of the National Academy of Science 2005102 (24) 8781-8788.

5. Pushpa S, Nallur B. Biological basis of dyslexia: A maturing perspective. Current Science 200690 (2) 168-175.

6. Sherman G. International Dyslexia Association Fact Sheet Nr 969 January 2000.

7. Pavlidis GT. Eye movements: The diagnostic key to dyslexia. S Afr Optom June/August 1988 29-39.

8. Evans B. The role of the optometrist in dyslexia: Part 1: Specific learning difficulties. Optometry Today January 30 2004 29-33.

9. Park GE. Reading Difficulty (dyslexia) from the ophthalmic point of view. American Journal of Ophthalmology 194831 (1) $28-34$.

10. Grigorenko EL. Developmental dyslexia: An update on genes, brains, and environments. Journal of Child Psychology and Psychiatry 200142 (1) 91-125.

11. Schumacher J, Hoffmann P, Schma C, Schulte G. Genetics of dyslexia: The evolving landscape. Journal of Medical Genetics 200744 289-297.
12. Snowling MJ. Specific learning difficulties. Psychiatry 20034 (9) 110-113.

13. Flax N. Problems in relating visual functions to reading disorders. American Journal of Optometry and Archives Academy of Optometry 197047 366-372.

14. Castro SM, Salgado CA, Andrade FP, Ciasca SM, Carvalho KM. Visual control in children with developmental dyslexia. Arq Bras Oftalmol. 2008 71(6) 837-40.

15. Evans BJ, Drasdo N, Richards IL. Investigation of some sensory and refractive visual factors in dyslexia. Vision Research 199434 (14) 1913-1926.

16. Buzelli AR. Stereopsis, Accommodative and Vergence Facility. Do they relate to dyslexia? Optometry and Vision Science 199168 (11) 842-846.

17. Goulandris L, McIntyre A, Snowling M, Bethel J, Lee J. A Comparison of dyslexic and normal readers using orthoptic assessment procedures. Dyslexia 19984 30-48.

18. Evans BJ, Drasdo N, Richards I. Investigation of accommodative and binocular function in dyslexia. Ophthalmic and Physiologic Optics 199414 5-18.

19. Helveston EM, Weber JC, Miller K. Visual function and academic performance. American Journal of Ophthalmology 198599 (3) 346-355.

20. Latvala ML, Korhoenen TT, Penttinen M. Ophthalmic findings in dyslexic school children. British Journal of Ophthalmology 199478 339-343.

21. Aasved H. Ophthalmological status of school children with dyslexia. Eye 1987 161-68.

22. Kapoula Z, Bucci M, Jurion F. Evidence for frequent divergence impairment in French dyslexic children: Deficit of convergence relaxation or of divergence per se? Graefe's Archives of Clinical Experimental Ophthalmology 2007 245 931-936.

23. Bucci M, Gignac D, Kapoula Z. Poor binocular coordination of saccades in dyslexic children. Graefes Archives of Clinical Experimental Ophthalmology 2008246 417-428.

24. Ygge J, Lennerstarnd G, Axelsson I, Rydberg A. Visual function in a Swedish population of dyslexic and normally reading children. Acta Ophtahlmologica (Copenh) 199371 (1) 1-9.

25. Eames TH. Comparison of eye conditions among 1000 reading failures, 500 ophthalmic patients and 150 unselected children. American Journal of Ophthalmology 194831 713-717.

26. Learning disabilities, dyslexia, and vision. Joint statement of American Academy of Ophthalmology, American Association for Pediatric Ophthalmology and Strabismus, American Association of Certified Orthoptists. Pediatrics 2009124 (2) 837-844.

27. Vision, learning and dyslexia. A Joint organizational policy statement of the American Academy of Optometry and the American Optometric Association. Optometry and Vision Science 199774 (10) 868-870.

28. Grisham JD, Simons HD. Refractive error and the reading process: A literature analysis. Journal of American Optometric Association 198657 (10) 44-55. 
29. Jamali P, Fotouhi A, Hashemi H, Younesian M, Jafari A. Refractive errors and amblyopia in children entering school: Shahrood, Iran. Optometry and Vision Science 200986 (4) 364-369.

30. Evans BJ. Visual factors in dyslexia. In: Turner M, Rack J. The Study of Dyslexia Kluwer Academic Press New York 2004 pp 1-22.

31. Eskridge JB, Amos, JF, Bartlett JD. Clinical Procedures in Optometry. JP Lippicott Company New York 1991.

32. Solan H. Learning Disabilities. In: Rosenbloom A, Morgan MW. Principles and Practice of Pediatric Optometry. JP Lippincott Publishers New York 1990.

33. Tong L Saw, Seang-Mei Carkeet Chan, Wai-Ying $\mathrm{Wu}$, Hui-Min Tan. Prevalence rates and epidemiological risk factors for astigmatism in Singapore school children. $O p$ tometry and Vision Science 200279 (9) 606-613.

34. Xue-Jiao Qin, Tom H Margrain, Chi Ho To, Nathan B, Guggenheim JA. Anisometropia is independently associated with both spherical and cylindrical ametropia. Investigative Ophthalmology and Vision Sciences $2005464024-$ 4031.

35. Doshi NR, Rodriguez, ML. Amblyopia. American Family Physician 200775 (3) 361-367.

36. Grosvenor T. Primary Care Optometry. Fifth Edition, Butterworth Heinemann Elsevier Philadelphia 2007.

37. Elliot D, Flanagan J, Hrynchak P, Prokopich L, Winn B. Clinical Procedures in Primary Eye Care. Second Edition. Butterworth Heinemann. Oxford 2003 pp 93-95, 126-127.

38. Morad Y, Lederman R, Avni I. Correlation between reading skills and different measurements of convergence amplitude. Current Eye Research 200225 (2) 117-121.

39. Owens DA, Wolf-Kelly K. Near work, visual fatigue, and variations of oculomotor tonus. Investigative Ophthalmology and Vision Sciences 198728 743-749.

40. Ygge J, Lennerstarnd G, Rydberg A, Wijecoon,S, Petterson BM. Oculomotor functions in a Swedish population of dyslexic and normally reading children. Acta Ophthalmologica (Copenh) 1993 71(1) 10-21.

41. Flax N. Visual function in dyslexia. American Journal of Optometry and Archive American Academy Optometry 196845 (9) 574-578.

42. Wick B, Hall P. Relation among accommodative facility, lag and amplitude in elementary school children. American Journal of Optometry and Physiological Optics 198764 (8) 593-598.

43. Allison C. "Eyedentify" your patients efficiency problems. Review of Optometry 2005142 (61) 1-10.

44. Evans BJ, Drasdo N. Review of ophthalmic factors in dyslexia. Ophthalmic and Physiologic Optics 199010 123132.

45. Suchoff IB. Research on the relationship between reading and vision-What Journal of Learning Disabilities 198114 (10) 573-576.

46. Stein JF, Riddell PM, Fowler S. Disordered vergence control in dyslexic children. British Journal of Ophthalmology 198872 162-166.

47. Wesson MD, Massen LC, Boyles ST. Objective testing of vergence ranges. Journal of American Optometric Association 199566 (6) 338-342. 